2018-05-08

\title{
A methodology for efficient code optimizations and memory management
}

Kelefouras, Vasileios

http://hdl.handle.net/10026.1/12670

10.1145/3203217.3203274

Proceedings of the 15th ACM International Conference on Computing Frontiers ACM

All content in PEARL is protected by copyright law. Author manuscripts are made available in accordance with publisher policies. Please cite only the published version using the details provided on the item record or document. In the absence of an open licence (e.g. Creative Commons), permissions for further reuse of content should be sought from the publisher or author. 


\section{A methodology for efficient code optimizations and memory management}

\author{
Vasilios Kelefouras \\ University of Leeds
}

\author{
Karim Djemame \\ University of Leeds
}

\begin{abstract}
The key to optimizing software is the correct choice, order as well parameters of optimizations-transformations, which has remained an open problem in compilation research for decades for various reasons. First, most of the compilation subproblems-transformations are interdependent and thus addressing them separately is not effective. Second, it is very hard to couple the transformation parameters to the processor architecture (e.g., cache size and associativity) and algorithm characteristics (e.g. data reuse); therefore compiler designers and researchers either do not take them into account at all or do it partly. Third, the search space (all different transformation parameters) is very large and thus searching is impractical.

In this paper, the above problems are addressed for data dominant affine loop kernels, delivering significant contributions. A novel methodology is presented that takes as input the underlying architecture details and algorithm characteristics and outputs the near-optimum parameters of six code optimizations in terms of either L1,L2,DDR accesses, execution time or energy consumption. The proposed methodology has been evaluated to both embedded and general purpose processors and for 6 well known algorithms, achieving high speedup as well energy consumption gain values over gec compiler, hand written optimized code and Polly.
\end{abstract}

\section{CCS CONCEPTS}

-Software and its engineering $\rightarrow$ Compilers; Retargetable compilers;

\section{KEYWORDS}

Code optimizations, data cache, register blocking, loop tiling, high performance, energy consumption, data reuse

\section{ACM Reference format:}

Vasilios Kelefouras and Karim Djemame. 2018. A methodology for efficient code optimizations and memory management. In Proceedings of CF '18: Computing Frontiers Conference, Ischia, Italy, May 8-10, 2018 (CF '18), 8 pages. DOI: $10.1145 / 3203217.3203274$

\section{INTRODUCTION}

Although significant advances have been made in developing advanced compiler optimization and code transformation frameworks, current compilers cannot compete with hand optimized code in terms of performance and energy consumption. Researchers tackle

Permission to make digital or hard copies of all or part of this work for personal or classroom use is granted without fee provided that copies are not made or distributed for profit or commercial advantage and that copies bear this notice and the full citation on the first page. Copyrights for components of this work owned by others than ACM must be honored. Abstracting with credit is permitted. To copy otherwise, or republish, to post on servers or to redistribute to lists, requires prior specific permission and/or a fee. Request permissions from permissions@acm.org.

CF '18, Ischia, Italy

(C) 2018 ACM. 978-1-4503-5761-6/18/05 ..\$15.00

DOI: $10.1145 / 3203217.3203274$ the code optimization problem by using heuristics [12], empirical techniques, iterative compilation techniques [11] and techniques that simultaneously optimize only two transformations, e.g., register allocation and instruction scheduling. The most promising approach is iterative compilation but is extremely expensive in terms of compilation time; therefore researchers and current compilers try to reduce compilation time by using i) both iterative compilation and machine learning compilation techniques [20], ii) both iterative compilation and genetic algorithms [11], iii) heuristics and empirical methods [5], iv) both iterative compilation and statistical techniques, v) exhaustive search [10]. However, by employing these approaches, the remaining search space is still so large that searching is impractical. The end result is that seeking the optimal configuration is impractical even by using modern supercomputers. This is evidenced by the fact that most of the iterative compilation methods use either low compilation time transformations only or high compilation time transformations with partial applicability so as to keep the compilation time in a reasonable level [9] [19] [13]. As a consequence, a very large number of solutions is not tested. This has led compiler researchers to use exploration prediction models focusing on beneficial areas of optimization search space [5]. Our approach differs in three main aspects. First, the transformations are addressed in a theoretical basis; second, together as one problem, and third by taking into account the Hardware (HW) architecture and algorithm characteristics.

The main steps of our methodology are as follows. First, we provide an efficient register blocking and loop tiling algorithm; these two algorithms consist of a) loop unroll, scalar replacement, register allocation and b) loop tiling, data array layout, transformations, respectively. A unified framework is proposed to orchestrate the aforementioned transformations, together as one problem (as they are interdependent); the transformations are tailored to the target processor architecture details and algorithm characteristics. Second, we make an analysis of how the above transformations affect Execution Time (ET) and Energy consumption (E) and for the first time we provide a theoretical model describing a) the number of L1 data cache (L1dc), L2 cache (L2c) and main memory (MM) accesses and b) the number of arithmetical instructions, as a function of the aforementioned transformation parameters, processor architecture details and algorithm input size; so, we are able to provide the transformation parameters giving a number of memory accesses close to the minimum. Third, taking advantage of this model, we make a first but important step towards correlating ET and E with the aforementioned transformation parameters, processor architecture details and algorithm input size.

The proposed methodology has resulted in five contributions, 1) a single framework addressing the aforementioned transformations theoretically but most importantly as one problem, 2) a new approach applying code optimizations (CO) by taking into account 
the HW architecture and the application special memory access patterns, 3) a theoretical model describing the number of memory accesses and arithmetical instructions, as a function of the aforementioned optimization parameters, HW architecture and algorithm input size, 4) a new approach correlating ET and Power consumption $(\mathrm{P})$ with the aforementioned transformation parameters, HW architecture and algorithm input size, 5) a direct outcome of contributions (1)-(4) is that the search space (to fine-tune the above optimizations) is reduced by many orders of magnitude.

The remainder of this paper is organized as follows. In Section 2, the related work is reviewed. The proposed methodology is presented in Section 3 while experimental results are discussed in Section 4. Finally, Section 5 is dedicated to conclusions.

\section{RELATED WORK}

Iterative compilation methods provide the most efficient approach towards the code optimization problem. However, to the best of our knowledge, there is no existing iterative compilation method including all the transformations presented in this paper and all different transformation parameters, because the compilation time becomes too large. Iterative compilation methods use either low compilation time transformations only or high compilation time transformations with partial applicability so as to keep the compilation time in a reasonable level [9] [19]. As a consequence, a very large number of solutions is not tested. In [19], loop tiling is applied with fixed tile sizes. In [9], multiple levels of tiling are applied but with fixed tile sizes. In [13], only loop unroll is applied.

[12] uses an artificial neural network to predict the best transformation (from a given set) should applied. In [5], performance counters are used to determine good compiler optimization settings. In [20], a long-term learning algorithm that determines the best set of heuristics is presented.

The polyhedral model is a flexible and expressive representation for loop transformations. In [17], a fundamental progress in the understanding of polyhedral loop nest optimizations is made. Polly is a high-level loop and data-locality optimizer and optimization infrastructure for LLVM [6]. Pluto, which is used by Polly, is an automatic parallelization tool based on the polyhedral model [4].

There has been significant research on reducing the number of data accesses in memory hierarchy by employing compiler transformations and most commonly loop tiling such as [4] [15]. In [15], a cache hierarchy aware tile scheduling algorithm for multicore architectures is presented.

Code optimizations are also used to reduce energy consumption in software. In [1], a survey about energy reduction methods is given. In [16], several transformation trade-offs are discussed. In [2], a compile-time approach to determine CPU frequency is proposed.

\section{PROPOSED METHODOLOGY}

In this paper, a novel methodology is presented that takes as input the underlying processor architecture and loop kernel characteristics and outputs the near-optimum parameters of the six aforementioned transformations in terms of either L1,L2,Main Memory (MM) accesses, Execution Time (ET) or Energy consumption (E).

Regarding target applications, this methodology considers affine loop kernels; it considers both perfectly and imperfectly nested loops, where all the array subscripts are linear equations of the iterators (which stands in most cases). This method is also applicable to loop kernels containing SIMD instructions. This method is applicable to all modern single-core and shared cache multi-core CPUs. Regarding shared cache processors, we use the software shared cache partitioning method given in our previous work [8]. No more than $p$ threads can run in parallel (one to each core), where $p$ is the number of the processing cores (single threaded codes only).

An abstract representation of our method is illustrated in Fig. 1 and it is further explained in the following Subsections.

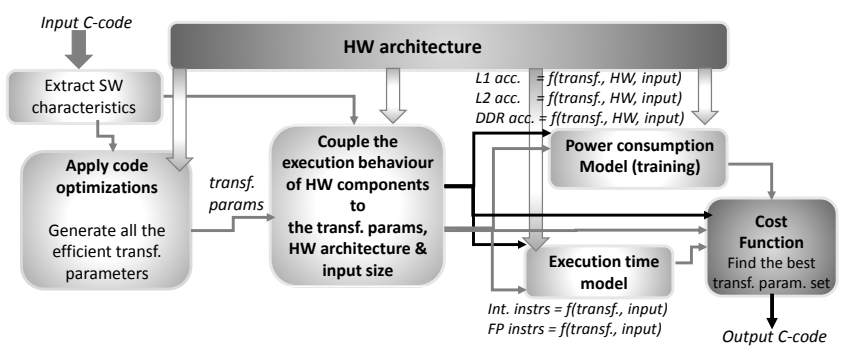

Figure 1: Flow chart of the proposed methodology

\subsection{Apply code optimizations}

In this Subsection we provide an efficient a) register blocking and b) loop tiling algorithm. The efficient application of loop tiling is not trivial and normally many different implementations are tested, since it a) depends on other transformations (e.g., data layout), b) depends on the target memory architecture and data reuse, c) increases the number of arithmetical instructions. The application of loop tiling for the Register File (RF) is even more complex (register blocking). To our knowledge, no application independent algorithm exists for register blocking; it is a mixture of loop tiling, loop unroll, scalar replacement and register allocation transformations. The above $\mathrm{CO}$ are the key to high performance and low energy consumption, especially for data dominant algorithms.

The main steps of the proposed register blocking algorithm are the following:

(1) Generate the subscript equations of all arrays

(2) Generate the RF inequality (Eq. 1) that provides all the efficient transformation parameters

(3) Extract a transformation set from Eq. 1

(4) Generate the code

Definition 3.1. Subscript equations which have more than one solution for at least one constant value, are named type2 equations. All others, are named type 1 equations.

For example, $(A[2 * i+j])$ gives the following type 2 equation $(2 * i+j=c 1)$, while $(A[i][j])$ gives the following type 1 equation $(i=c 21$ and $j=c 22)$.

Each subscript equation defines the memory access pattern of the specific array reference. Obviously, in our methodology type 1 and type 2 arrays are treated with different policies as they access data in different ways.

The RF inequality (Eq. 1) gives the exact loops that loop unroll is applied to, their unroll factor values and the number of variables/registers allocated for each array. Each subscript equation contributes to the creation of Eq. 1, i.e., equation $i$ gives $A r_{i}$ and 
specifies its expression. The implementations that do not obey to the extracted inequalities are discarded reducing substantially the search space. The RF inequality is given by

$$
n+S c \leq A r_{1}+A r_{2}+\ldots+A r_{n}+S c \leq F P
$$

where $F P$ is the number of the floating point (FP) registers, $S c$ is the number of FP scalar variables and $n$ is the number of the array references. Without any loss of generality, we assume that the arrays contain FP data only; in this case, the number of integer variables used is always smaller than the number of integer registers. The upper bound of Eq. 1 derives from the fact that if more registers than the available are used, data are spilled to L1dc, increasing the number of $\mathrm{L} 1$ accesses. On the other hand, the lower bound value is small because other constraints may be more critical. By using a larger lower bound value, register utilization is increased and therefore the number of L1 accesses is reduced; however, these transformation parameters may conflict to those minimizing the number of MM or L2 accesses, which may be more critical.

The number of variables/registers allocated for every array is given by both Eq. 2 and the three bullets below (the bullet points are given in order to assign variables according to data reuse).

$$
A r_{i}=u n r_{-} 1^{\prime} \times u n r_{-} 2^{\prime} \times \ldots \times u n r_{-} n^{\prime}
$$

where the integer $u n r_{-} i^{\prime}$ are the unroll factor values of the iterators exist in the array's subscript, e.g., the $C[i][j]$ array in Fig. 2 gives $\left(A r_{C}=1 \times 4=4\right)(r 1-r 4$ variables $)$ as the $(i, j)$ iterator unroll factor values are $(1,4)$, respectively.

- For the type1 arrays which contain all the loop kernel iterators, only one register is needed $\left(A r_{i}=1\right)$

- For the innermost iterator always holds $u n r^{\prime}=1$

- For the arrays i) containing more than one iterators and one of them is the innermost and ii) all iterators which do not exist in this array reference have unroll factor values equal to 1 , then only one register is needed for this array $\left(A r_{i}=1\right)$

In the above three cases, a different element is accessed in each iteration (no data reuse being achieved) and thus wasting more than one register is not efficient, e.g., in Fig. 2, six registers are used, i.e., $\left(A r_{C}=1 \times 4, A r_{A}=1 \times 1, A r_{B}=1\right)$. Note that $\left(A r_{B}=1\right)$ instead of $\left(A r_{B}=1 \times 4\right)$ because of the 3rd bullet above (a different element of $B$ is accessed in each $k$ iteration and therefore it is not efficient to waste more than one register).

Let us give an example, first box code in Fig. 2. Eq. 1 gives:

$3 \leq u n r_{i} \times u n r_{j}+u n r_{i}+u n r_{j} \leq F P, u n r_{i} \neq 1 \& u n r_{j} \neq 1$

$$
\begin{aligned}
& 3 \leq u n r_{j}+2 \leq F P, u n r_{i}=1 \\
& 3 \leq u n r_{i}+2 \leq F P, u n r_{j}=1
\end{aligned}
$$

The 3 rd bullet generates 3 branches while the 2 nd gives $\left(u n r_{k}=\right.$ 1). The code shown in the second box of Fig. 2 refers to a second branch solution, i.e., $\left(u n r_{i}=1\right.$ and $\left.u n r_{j}=4\right)$ and therefore 6 registers are used.

The main steps of the loop tiling algorithm are similar to those of the register blocking algorithm, but a cache inequality (Eq. 4) is generated for each cache memory; each inequality contains the iterators that loop tiling is applied to, the tile sizes and the data array layouts. The implementations that do not obey to the extracted inequalities are automatically discarded by our methodology reducing substantially the search space.

The cache inequality is formulated as:



Figure 2: An example, Matrix-Matrix Multiplication (MMM)

$$
m \leq\left\lceil\frac{\text { Tile }_{1}}{L_{i} / \text { assoc }}\right\rceil+\ldots+\left\lceil\frac{\text { Tile }_{n}}{L_{i} / \text { assoc }}\right\rceil \leq \text { assoc }
$$

where $L_{i}$ is the corresponding cache size, assoc is the $L_{i}$ associativity value (e.g., for an 8-way associative cache, assoc $=8$ ) and $m$ defines the lower bound of the tile sizes and it equals to the number of arrays in the loop kernel. In the special case where the number of the arrays is larger than the associativity value is not discussed in this paper (normally, $($ assoc $\geq 8)$ ). Tile $i$ gives the tile size of the ith array and is given by Eq. 5 :

$$
\text { Tile }_{i}=T_{1}^{\prime} \times T_{2}^{\prime} \times T_{n}^{\prime} \times \text { type } \times s
$$

where type is the size of each array's element in bytes and $T_{i}^{\prime}$ are the tile sizes of the iterators existing in the corresponding array subscript. $s$ is an integer and $(s=1$ or $s=2)$; $s$ defines how many tiles of each array should be allocated in the cache. For the tiles that do not achieve data reuse (a different tile is accessed in each iteration), we assign cache space twice the size of their tiles ( $s=2$ in Eq. 5). This way, not one but two consecutive tiles are allocated into the cache in order for the second accessed tile not to displace another array's tile. $\left(\left\lceil\frac{\text { Tile }_{1}}{L_{i} / \text { assoc }}\right\rceil\right)$ is an integer representing the number of $L_{i}$ cache lines with identical cache addresses used for the tile of array1. Eq. 4 satisfies that the array tiles directed to the same cache subregions do not conflict with each other as the number of cache lines with identical addresses needed for the tiles is not larger than the (assoc) value.

All the tile elements in Eq. 4 must contain consecutive MM locations, e.g., in Fig. 2, none of the tiles does. Otherwise, an extra loop kernel is added for each array, likewise Atr and Btr arrays in Fig. 2; new arrays are created which replace the default ones (extra cost in L/S and arithmetical instructions). There are some special cases where the arrays do not contain consecutive memory locations but their layouts can remain unchanged in order to avoid the cost of transforming the arrays; in that case, extra 
cache misses occur and thus a larger error in approximating the number of memory accesses occurs too (Fig. 4-Subsection 4.1). $T_{i}^{\prime}$ is given by one of the following three:

- $T_{i}^{\prime}$ equals to the L1 tile size of $i$ iterator, if tiling for L1 is applied to the i iterator

- $T_{i}^{\prime}$ equals to the unroll factor value of $i$ iterator, if tiling for L1 is not applied to the i iterator and i has a smaller Nesting Level Value (NLV) than the iterator being tiled for L1

- $T_{i}^{\prime}$ equals to the upper loop bound value of $\mathrm{i}$ iterator, if tiling for L1 is not applied to the i iterator and i has a larger NLV than the iterator being tiled for L1

Assuming an 8-way 32kbyte L1dc and MMM (Fig. 2), Eq. 4 gives $\left(3 \leq\left\lceil\frac{T_{C 1}}{4096}\right\rceil+\left\lceil\frac{T_{A 1}}{4096}\right\rceil+\left\lceil\frac{T_{B 1}}{4096}\right\rceil \leq 8\right)$. The $\left(T_{C 1}, T_{A 1}, T_{B 1}\right)$ values of the $\mathrm{C}$-code shown at the right of Fig. 2 are given in the bottom left box (the NLV of $k$ iterator is 6 while the NLV of $k k$ is 1 ), also, floating point values are assumed, 4 bytes each.

In the shared cache case, $L_{i}$ in Eq. 4 is the corresponding shared cache partition size used and each core uses only its assigned shared cache space.

We have implemented an automated $C$ to $C$ tool just for the six studied algorithms, but a general tool can be implemented by using POET [18] tool.

\subsection{Couple execution behaviour to $\mathrm{CO}$, processor architecture $\&$ input size}

For all the Eq. 1 and Eq. 4 schedules, we compute the number of L1dc, L2c and MM accesses as well as the number of arithmetical instructions. This problem is theoretically formulated by exploiting the memory architecture details and the special memory access patterns. In particular, one mathematical equation is generated for each memory and for each loop kernel providing the corresponding value. This equation provides the number of memory accesses while the transformation parameters and input size serving as the independent variables of the equation. Loop tiling and loop unroll transformations as well as the input size, are inserted directly to the aforementioned equations while the data layouts, scalar replacement and register allocation transformations as well as the HW architecture, are inserted indirectly (they have been used in order to create Eq.1-Eq.5). This way, we are able to find the solution offering a number of L1dc, L2c or MM accesses close to the minimum.

We are able to approximate the number of memory accesses because no unexpected misses occur in the cache. We assume that the underlying memory architecture consists of separate first level data and instruction caches (modern architectures). In this case, the program code typically fits in L1 instruction cache; thus, it is assumed that the shared cache or unified cache (if any) is dominated by data. For the reminder of this paper, we assume 2 levels of cache, but more/less levels can be used, by slightly changing the following equations.

The equation approximating the number of L1dc accesses follows L1.acc $=\sum_{i=1}^{i=\text { arrays }}\left(\prod_{j=1}^{j=M} \frac{\left(u p_{j}-\text { low }_{j}\right)}{T_{j}} \times \prod_{k=1}^{k=P} u n r_{k}+\right.$ off $_{j}$ set $\left._{i}\right)+$ var

where arrays is the number of arrays, $M$ is the number of the iterators that control the corresponding array and $P$ is the number of the iterators that loop unroll has been applied to (iterators that exist in the subscript of the corresponding array only), e.g., regarding the $C$ array in the code at the right of Fig. 2, the first product of Eq. 6 refers to all the iterators but $k$ (array reference is outside $k$ loop) while the second product refers to $j$ iterator only. (up, low) give the bound values of the corresponding iterator (normally, they define the algorithm's input size) and $(T, u n r)$ refer to the tile size and unroll factor value, respectively.

of f set gives the number of L1dc of the new loop kernel added in the case the data array layout is transformed. Offset is either $\left(\right.$ offset $_{i}=2 \times$ ArraySize $\left._{i}\right)$ or $\left(\right.$ off set $\left._{i}=0\right)$ depending on whether the data layout of the array is changed or not; in the case that the layout is changed, the array has to be loaded and then written again to memory, thus it is $\left(\right.$ off set $i=2 \times$ ArraySize $\left._{i}\right)$. (var) gives the number of L1 accesses due to the scalar variables; we never use more registers than available and thus the number of RF spills is negligible (var $\approx 0$ ).

Eq. 6 for the C-code at the right of Fig. 2 gives $\left(\frac{N^{3}}{4 \times K K}, \frac{N^{3}}{4}, N^{3}\right) \mathrm{L} 1$ accesses for $(C, A, B)$ arrays, respectively, and in overall $(L 1 . a c c=$ $\left.\frac{N^{3}}{4 \times K K}+\frac{N^{3}}{4}+N^{3}+4 \times N^{2}\right)$. Here, the number of L1 accesses strongly depends on the unroll factor value $\left(N^{3} / 4\right)$.

The number of L2c accesses is approximated by Eq. 7; at this step, only the new/extra iterators (introduced by loop tiling) must be processed and not the initial iterators exist in the input code.

L2_Acc. $=\sum_{i=1}^{i=t y p e 1}$ Type1_L2acc. $+\sum_{i=1}^{i=t y p e 2}$ Type2_L2acc. + code

where type 1 and type 2 is the number of type 1 and type 2 arrays, respectively. In this paper, we don't provide the equations for type 2 arrays because of the limited paper size; however, in Section 4, FIR and Gaussian Blur contain type2 arrays. code refers to the number of source code accesses and always (Arrays_acc. $\gg$ code) as a) the code size of loop kernels is small and fits in L1 instruction cache, b) we are dealing with data dominant algorithms.

$$
\text { Type } 1 \text { L2acc. }=\text { array_size } \times t i+\text { of } f \text { set }
$$

where array_size is the size of the array and of $f$ set gives the number of L2 accesses of the new loop kernel added in the case the data array layout is transformed. $t_{i}$ gives how many times the corresponding array is accessed from L2 memory and is given by Eq. 9. Regarding the of $f$ set value, when the array size is bigger or comparable to the cache size, then (offset $i>2 \times$ ArraySize $\left._{i}\right)$. This is because the elements are always loaded in blocks (cache lines) and many lines are loaded more than once (especially in the column-wise case). This is why we use a hand optimized code changing the layout in an efficient way, thus always achieving (offset of $_{i} \approx 2 \times$ ArraySize $_{i}$ ).

$$
t i=\prod_{j=1}^{j=N} \frac{\left(u p_{j}-l o w_{j}\right)}{T_{j}} \times \prod_{k=1}^{k=M} \frac{\left(u p_{k}-l o w_{k}\right)}{T_{k}}
$$

where $N$ is the number of new/extra iterators that a) do not exist in the corresponding array and b) exist above of the iterators of the corresponding array. $M$ is the number of new/extra iterators that a) do not exist in the array and $b$ ) exist between of the iterators of the array, e.g., regarding $(C, A, B)$ arrays in Fig. 2 , the iterators referring to the first and second product of Eq. 9 are $(k k, n o n e)$, (jj, none), (none, ii), respectively, giving $\left(t_{C}=\frac{N}{K K}\right),\left(t_{A}=\frac{N}{J J}\right)$ and $\left(t_{B}=\frac{N}{I I}\right)$, respectively. The first and the second products of Eq. 9 give how many times the array is accessed due to the iterators exist above the upper new iterator of this array and between the new iterators of this array, respectively. 
Eq. 7 for the code of Fig. 2 gives $\left(L 2 . a c c=N^{3} / K K+N^{3} / J J+\right.$ $N^{3} / I I+4 \times N^{2}$ ).

In the case that more than one thread run in parallel under a shared cache, the overall number of cache accesses is extracted by accumulating all the different loop kernel equations.

The number of MM accesses is given by an equation identical to Eq. 7. Moreover, the number of MM accesses because of the type1 arrays is given by an equation identical to Eq. 8 and Eq. 9. However, in Eq. 9, we refer only to the iterators created by applying tiling to the last level cache, e.g., regarding $(C, A, B)$ arrays of MMM (Fig. 2), the iterators referring to the first and second product of Eq. 9 are ( $k k$, none), (none, none), (none, $i i)$, respectively, giving $\left(t_{C}=\frac{N}{K K}\right)$, $\left(t_{A}=1\right)$ and $\left(t_{B}=\frac{N}{I I}\right)$, respectively.

The number of integer and FP instructions is approximated by:

$$
\text { Arith._instrs }=\sum_{i=1}^{i=i \text { terators }}\left(\prod_{j=1}^{j=i} \frac{u p_{j}-l o w_{j}}{T_{j}} \times c_{j}\right)+\text { offset }
$$

where iterators is the total number of iterators and (up, low, T) are their corresponding bound values, as in previous equations. $c_{j}$ is the number of integer or FP assembly instructions measured inside $j$ loop (assembly instructions occur between the open and close loop bracket). of $f$ set is the number of arithmetical instructions of the extra loop kernels added (if the array layouts change).

$\left(\sum_{i=1}^{i=i \text { terators }}\left(\prod_{j=1}^{j=i} \frac{u p_{j}-\text { low }_{j}}{T_{j}}\right)\right.$ gives the number of loop iterations in total while $c_{j}$ gives the number of assembly instructions in loop $j$. Note that $j$ iterator varies from $(j=1$ - it corresponds to the outermost iterator) to $(j=$ iterators - it corresponds to the innermost iterator), e.g., in Fig. 2, Eq. 10 gives $((N / K K) \times c 1+$ $\left(N^{2} /(K K \times I I)\right) \times c 2+\left(N^{3} /(K K \times I I \times J J)\right) \times c 3+\left(N^{3} /(K K \times J J)\right) \times$ $\left.c 4+\left(N^{3} /(K K \times 4)\right) \times c 5+\left(N^{3} / 4\right) \times c 6\right)$; as it can be observed, the number of arithmetical instructions is strongly affected by a) the number of the loops being tiled (more terms are introduced), b) tile size / unroll factor values of the innermost iterators (here, the unroll factor value of $j$, i.e., 4 , affects the number of instructions at the most). Thus, a larger unroll factor value would be more efficient.

Given that the $c$ values depend on the target compiler, they cannot be approximated. Thus, we measure the $c$ values for one transformation set and predict the $c$ values of the others (where possible), e.g., in Fig. 2, the $c$ values (assembly instructions) almost remain unchanged by changing the $(K K, I I, J J)$ values (apart from their maximum and minimum ones because in this case, the number of the loops changes), but not by changing the $(j)$ unroll factor value or the number of the loops being tiled, because the loop body changes and thus more/less assembly instructions are inserted.

In this work, we take advantage of the fact that the $c$ values almost remain unchanged for different tile sizes, suffice the array layouts remain unchanged and the tile sizes do not take their maximum/minimum values; the $c$ values are only slightly affected by the compiler, even by using aggressive compilers and high optimization levels. The $c$ values for different unroll factor values and data layouts are significantly changed and cannot be predicted.

\subsection{Performance Models}

The aforementioned transformations affect $\mathrm{P}$ and $\mathrm{E}$ in all $\mathrm{HW}$ components and thus a different power model is generated for each different processor and MM. An off-line training phase is applied in order to generate the power equations for the target processor and MM (Fig. 3); the custom HW architecture is given as input to the

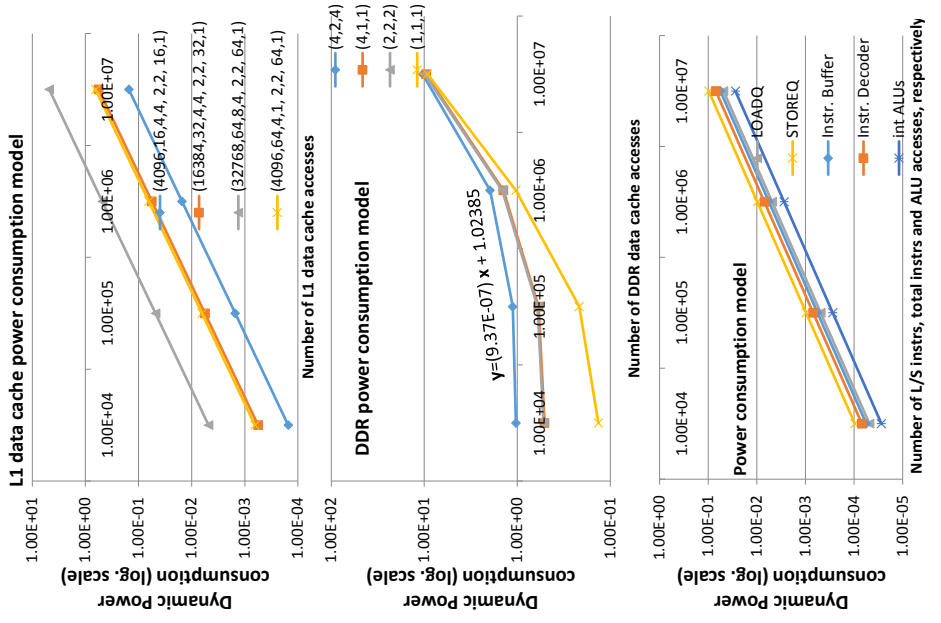

Figure 3: Power consumption models

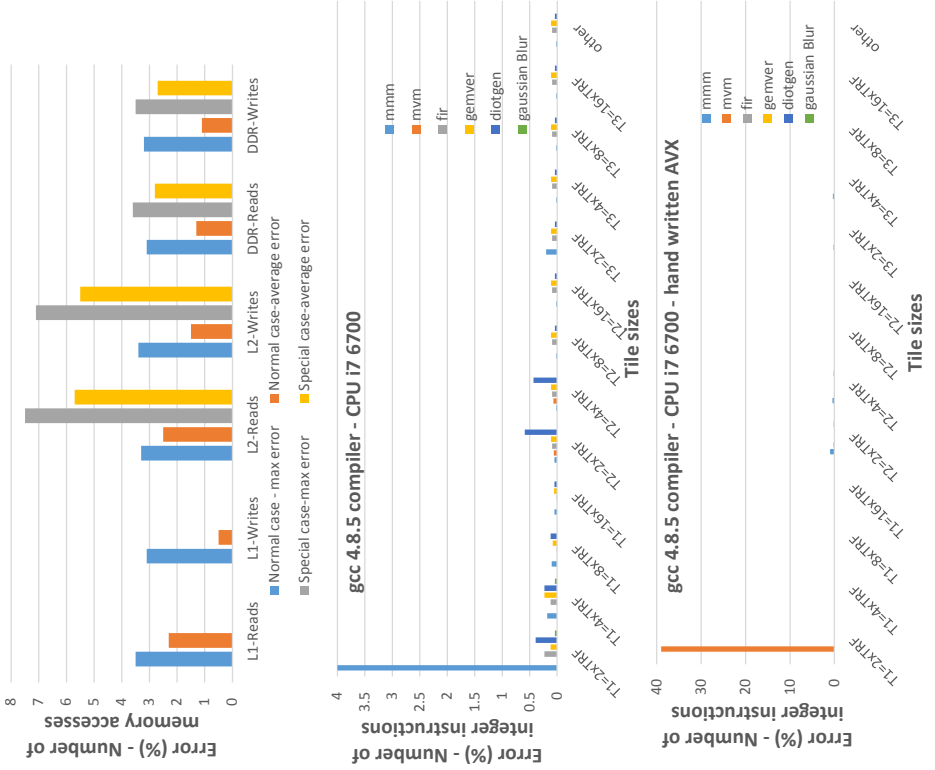

Figure 4: Validation of Eq.6-Eq.10 (relative error)

mcpat [14] simulator and a number of simulations takes place for different values of L1,L2,DDR accesses and integer, FP instructions. This way we generate $\mathrm{P}$ equations (Fig. 3); we show that memory power values are linear to their number of memory accesses (Eq.6Eq.10). Moreover, LoadQ/StoreQ power values are linear to the number of L/S instructions (Eq. 6). The ALU, instruction buffer and instruction decoder power values are linear to the number of ALU instructions and total number of instructions (Eq. 10+Eq. 6), respectively (Fig. 3). Although this work can be extended to take into account more HW architecture components, in this paper, we approximate $\mathrm{P}$ by using Eq. 11; thus, according to mcpat we do not take into account $\mathrm{P}$ on the renaming unit, instruction cache, $\mathrm{RF}$, TLBs, branch predictor and instruction scheduler. 


$$
\begin{array}{r}
P=P_{L 1}(f(\text { L1.acc }))+P_{L 2}(f(L 2 . a c c))+P_{D D R}(f(D D R . a c c))+ \\
P_{L / S \_Q u e u e}(f(L / \text { S.instrs }))+P_{A L U}(f(\text { ALU.instrs }))+ \\
P_{\text {instr.buffer }}(f(\text { instrs }))+P_{\text {instr.decoder }}(f(\text { instrs }))
\end{array}
$$

As far as execution time (ET) is concerned, it cannot be approximated by using a mathematical formula; however, we can use current ET models in order to find/predict qualitatively the transformation parameter set giving the fastest binary. Given that all the candidate transformation parameter sets a) refer to the same algorithm, b) the algorithm is static, in Subsection 4.1, we show that we are able to select qualitatively a high quality transformation parameter set, even by using a simple execution time model (Average Memory Access Time (AMAT) [7]). Although more accurate and complex ET models exist like [22], where concurrency in memory hierarchy is taken in account, the aim of this first version is to validate and describe the theoretical background.
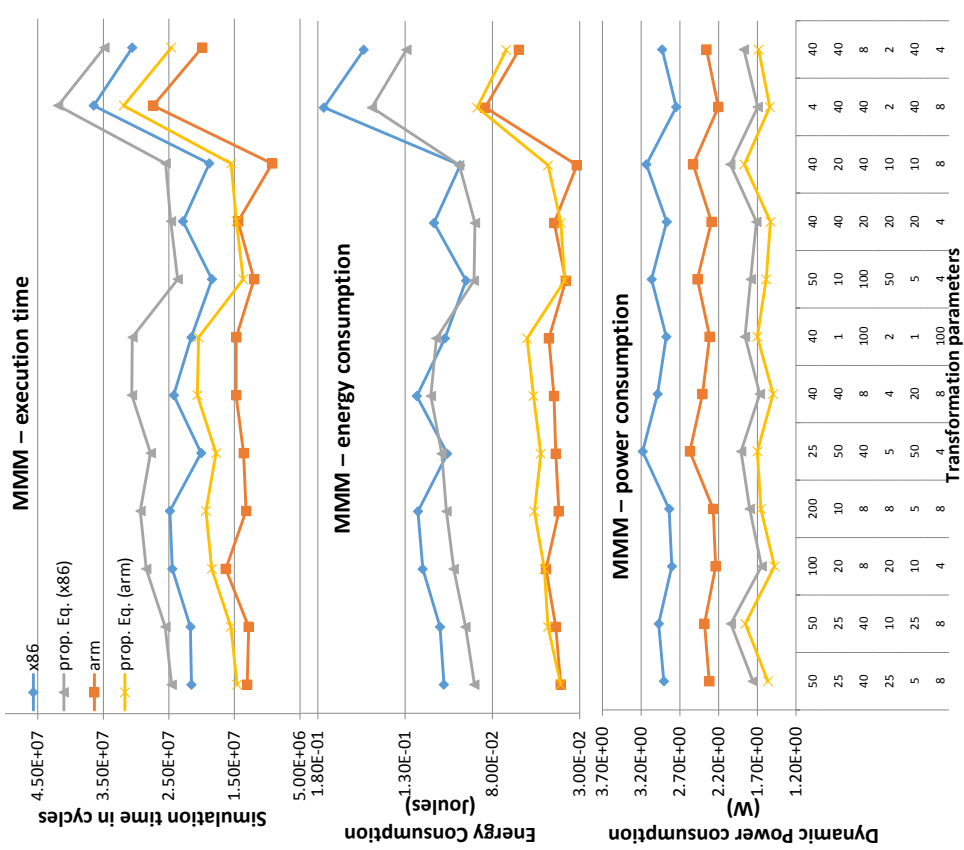

Figure 5: Validation of the ET and $P$ models used (the results for the other algorithms are similar)

\subsection{Reduction of the search space - optimality}

Although it is impractical to run all different schedules in order to prove that our methodology doesn't discard efficient schedules, a theoretical explanation is given.

First, the schedules that don't belong to Eq. 1, either use a larger number of registers than available or they don't take into account data reuse (and therefore registers are wasted), while the schedules that don't belong to Eq. 4 either use larger tile sizes than the cache or the tiles cannot remain in the cache. All the above refer to schedules that register blocking and loop tiling have not been applied in an efficient way and therefore they give a high number of data accesses through the whole memory hierarchy. Although Eq. 1 and Eq. 4 transformation parameters do not always provide near-optimum performance as the corresponding transformations are not always efficient/desirable, Eq. 1 and Eq. 4 do provide all the efficient register blocking and loop tiling implementations, respectively. In other words, if the target metric is to minimize the number of $L_{i}$ memory, then the optimum solution will be included in the corresponding inequality.

\subsection{Putting it all together}

The proposed methodology is given in Algorithm 1. All the steps have been explained in the previous subsections. All different combinations of loop interchange are generated as it affects Eq.1Eq.10.

In the case that the target metric is not ET or E, but the minimum number of $L_{i}$ memory accesses, then Algorithm 1 is changed accordingly, i.e., steps $(1,2,5,8),(1,3,5,8)$ or $(1,4,5,8)$ are executed only, respectively. It is important to note that in this case the number of different schedules that have to be further processed by Subsection 3.2 is smaller, i.e., the lower bound values of Eq. 1 and Eq. 4 are no longer needed to be that small. For example, by using a larger lower bound value in Eq. 1, register utilization is increased and therefore the number of L1 accesses is reduced; however, these parameters may conflict to those minimizing the number of MM accesses, which may be more critical. Thus, if the target metric is just the L1dc accesses, there is no need to use such a small Eq. 1 lower bound value. The same holds for L2c and MM too.

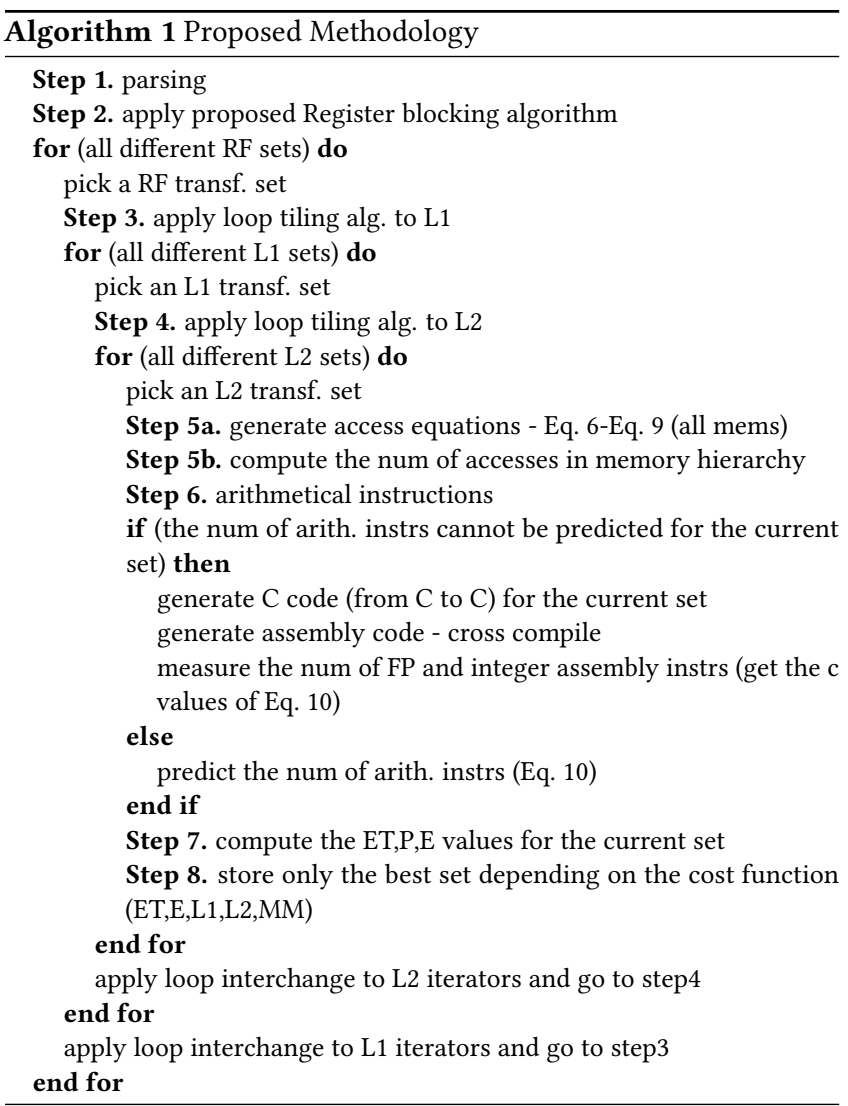

\section{EXPERIMENTAL RESULTS}

The experimental results are obtained by using a) the quad-core Intel i7 6700 CPU (CentoS-7 OS) by using both normal C-code and hand 
Table 1: Evaluation over gec and hand written AVX code

\begin{tabular}{|c|c|c|c|c|c|c|}
\hline \multirow{5}{*}{$\begin{array}{c}\text { Binaries } \\
\text { default } \\
\text { best ET } \\
\text { best E }\end{array}$} & \multicolumn{3}{|c|}{ MMM - ZYBO } & \multicolumn{3}{|c|}{ MVM - ZYBO } \\
\hline & ET(sec) & $\mathbf{P}(\mathbf{W})$ & $E(J)$ & ET( & $\mathbf{P}(\mathbf{W})$ & $E(J)$ \\
\hline & & & & & & \\
\hline & & & & & & \\
\hline & & 3.80 & & & & \\
\hline & \multicolumn{3}{|c|}{ MMM - i7 6700} & \multicolumn{3}{|c|}{ MVM - i7 6700} \\
\hline default & $9.92 \mathrm{E}+00$ & $4.80 \mathrm{E}+01$ & $4.76 \mathrm{E}+02$ & $\mathrm{E}-01$ & $4.18 \mathrm{E}+01$ & +00 \\
\hline & 00 & $46 t-2 x$ & & & & \\
\hline Dest a 1 & +00 & 4.0 & -02 & & & \\
\hline \multirow[t]{2}{*}{ best $\mathrm{E}$} & & 4.3 & & & & \\
\hline & \multicolumn{3}{|c|}{ FIR - ZYBO } & \multicolumn{3}{|c|}{ Diotgen - ZYBO } \\
\hline & & $5.75 \mathrm{E}-01$ & 0 & +01 & & 1.9 \\
\hline & 8.2 & 5.0 & & & & \\
\hline \multirow[t]{2}{*}{ best $\mathrm{E}$} & & 4.75 & & & & \\
\hline & \multicolumn{3}{|c|}{ FIR - i7 6700} & \multicolumn{3}{|c|}{ Diotgen - i7 6700} \\
\hline & & & & & & 4.28 \\
\hline & 1.50 & 01 & & & & +02 \\
\hline bes & 02 & 01 & & 0 & & +02 \\
\hline \multirow[t]{2}{*}{ best $\mathrm{E}$} & & $4.70 \mathrm{E}+01$ & & $.38 \mathrm{E}+00$ & $4.55 \mathrm{E}+01$ & $1.08 \mathrm{E}+02$ \\
\hline & \multicolumn{3}{|c|}{ Gemver - ZYBO } & \multicolumn{3}{|c|}{ Gaussian Blur - ZYBO } \\
\hline & $4.90 \mathrm{E}-01$ & $5.75 \mathrm{E}-01$ & $2.82 \mathrm{E}-01$ & $2.53 \mathrm{E}-01$ & & $1.04 \mathrm{E}-01$ \\
\hline & $2.10 \mathrm{E}-01$ & $5.00 \mathrm{E}-01$ & $1.05 \mathrm{E}-01$ & $6.65 \mathrm{E}-02$ & & $2.33 \mathrm{E}-02$ \\
\hline \multirow[t]{2}{*}{ best $\mathrm{E}$} & 10E-01 & $4.75 \mathrm{E}-01$ & -02 & $6.65 \mathrm{E}-02$ & $3.50 \mathrm{E}-01$ & $2.33 \mathrm{E}-02$ \\
\hline & \multicolumn{3}{|c|}{ Gemver - i7 6700} & \multicolumn{3}{|c|}{ Gaussian Blur - i7 6700} \\
\hline & -03 & $4.47 \mathrm{E}+01$ & $9.39 \mathrm{E}-02$ & $2.60 \mathrm{E}-02$ & $4.62 \mathrm{E}+01$ & $1.20 \mathrm{E}+00$ \\
\hline & .00 & & & & & $2.85 \mathrm{E}-01$ \\
\hline & $1.54 \mathrm{E}-03$ & $3.65 \mathrm{E}+01$ & $5.62 \mathrm{E}-02$ & $5.40 \mathrm{E}-03$ & $4.64 \mathrm{E}+01$ & $2.51 \mathrm{E}-01$ \\
\hline best $\mathrm{E}$ & $1.54 \mathrm{E}-03$ & $3.65 \mathrm{E}+01$ & $5.62 \mathrm{E}-02$ & $5.40 \mathrm{E}-03$ & $4.64 \mathrm{E}+01$ & $2.51 \mathrm{E}-01$ \\
\hline
\end{tabular}

written code with AVX extensions, b) the embedded ARM CortexA9 processor on a Zybo Zynq-7000 FPGA platform using petalinux OS, c) the gem5 [3] and McPAT [14] simulators, simulating both a generic $\mathrm{x} 86$ and an ARMv8-A CPU .

The bench-suite used in this study consists of six well-known data dominant static kernels taken from PolyBench/C benchmark suite version 3.2 [21]. These are: Matrix-Matrix Multiplication (MMM), Matrix-Vector Multiplication (MVM), Gaussian Blur $(3 \times 3$ filter), Finite Impulse Response filter (FIR), a kernel containing mixed vector multiplication and matrix addition (Gemver) and a multiresolution analysis kernel (Diotgen). The kernels are compiled using gcc 4.8.5 and arm-linux-gnueabi-gcc 4.9.2 compilers, for $\mathrm{x} 86$ and arm, respectively ('O3'). The output of our method is compiled with 'O2' optimization level in order the compiler to be less aggressive.

Table 2: Speedup over hand optimized code and Polly

$\begin{array}{ccccccc} & \begin{array}{c}\text { Unroll } \\ \text { 1 loop }\end{array} & \begin{array}{c}\text { Tiling } \\ \text { 1 loop }\end{array} & \begin{array}{c}\text { Tiling } \\ \text { 2 loops }\end{array} & \begin{array}{c}\text { Unroll \& } \\ \text { Tiling }\end{array} & \begin{array}{c}\text { Prop. } \\ \text { Meth. }\end{array} & \begin{array}{c}\text { Polly } \\ \text { LLVM }\end{array} \\ \text { MMM-i7(AVX) } & 1.11 & 1.53 & 1.82 & 1.90 & 3.93 & 1.41 \\ \text { MMM-ZYBO } & 1.71 & 2.23 & 2.78 & 3.07 & 8.62 & \\ \text { MVM-i7(AVX) } & 1.08 & 1.09 & 1.09 & 1.10 & 2.32 & 0.97 \\ \text { MVM-ZYBO } & 1.18 & 1.11 & 1.10 & 1.13 & 2.14 & \\ \text { FIR-i7(AVX) } & 1.42 & 1.11 & 1.11 & 1.44 & 3.85 & 1.38 \\ \text { FIR-ZYBO } & 1.31 & 1.52 & 1.50 & 1.63 & 2.48 & \\ \text { Gemver-i7(AVX) } & 1.06 & 1.03 & 1.03 & 1.07 & 1.26 & 1.31 \\ \text { Gemver-ZYBO } & 1.33 & 1.04 & 1.04 & 1.35 & 2.34 & \\ \text { Diotgen-i7(AVX) } & 1.16 & 1.53 & 1.60 & 1.65 & 3.91 & 1.26 \\ \text { Diotgen-ZYBO } & 1.34 & 2.69 & 3.05 & 3.38 & 30.63 & \\ \text { G.Blur-i7(AVX) } & 1.02 & 1.00 & 1.00 & 1.02 & 1.17 & 1.02 \\ \text { G.Blur-ZYBO } & 1.62 & 1.00 & 1.00 & 1.62 & 3.81 & \end{array}$

\subsection{Validation of Eq.6-Eq.10 (gem5)}

First, a validation on the number of arithmetical instructions is given (Eq. 10) for 2 different compilers ('O2' option). The number of integer instructions is measured for one transformation set and then predicted for the others (2nd and 3rd figure in Fig. 4); we take advantage of the fact that the $c$ values almost remain unchanged for different tile sizes. In Fig. 4, (T1,TRF) represent the (tile, unroll factor) values of the innermost iterator, respectively, $(T 2, T R F)$ the next innermost etc. There is a large error value in the case that the tile size of the innermost iterator is twice its minimum value (this is the only case we have faced this disunion); these parameter sets are not efficient in the majority of the cases and this is why the compiler becomes so aggressive and therefore changes the code. It is important to note that this disunion on the error values is eliminated by using the 'O1' option. Thus, in order to use 'O2' option, the $(T 1=2 \times T R F)$ case has to be included in Step6 of Algorithm 1. The results on the FP instructions are similar.

Subsection 3.2 has also been validated on the number of L1, L2 and MM accesses (1st figure in Fig. 4). The error values are less than $3.5 \%$ in all cases (both processors) when the tiles contain consecutive MM locations. However, as it was expected, for the special cases that the array layouts remain unchanged, there is a larger error.

\subsection{Validation of execution time and power consumption models (gem5 and McPAT)}

Furthermore, a validation on the simple ET model used [7] is made as well as on the $\mathrm{P}$ model derived by mcpat (Fig. 5). The equations giving the execution time for the $\mathrm{x} 86$ and arm on Gem5 are $(E T=$ L1reads $* 2+$ L2reads $* 20+$ DDRreads $* 60)$ and $(E T=$ L1reads + L1writes + L2reads $* 20+$ DDRreads $* 60)$, respectively. These equations don't take into account the concurrency in memory hierarchy and this is why the equations give larger ET values in most cases. However, the above simple equations give very good results because a) all different transformation parameters refer to the same algorithm, b) the algorithms are static; apart from not taking into account concurrency in memory hierarchy, any less accurate measurements derive from the fact that the above ET equations do not take into account instruction level parallelism. The reason that arm processor achieves less execution cycles than $\mathrm{x} 86$ in gem 5 simulator is that a) unlike $\mathrm{x} 86$, arm compiler generates assembly code with fused multiply-accumulate assembly instructions, b) x86 contains more registers than arm and thus the current transformation sets are more efficient for arm. Regarding $\mathrm{P}$, the proposed model follows perfectly the trend in both arm and $\mathrm{x} 86$, but $\mathrm{P}$ is more accurate on arm than on $\mathrm{x} 86$; $\mathrm{x} 86$ is more complex than arm and therefore the HW components that we have not taken into account consume more power.

\subsection{Evaluation over gec, hand tuning optimized code \& Polly (i7 6700 \& ARMv8-A)}

First, the proposed methodology is evaluated over gcc compiler on two real processors (Table 1). Intel i7 processor supports SIMD unit and therefore we have evaluated our methodology to C-code containing AVX intrinsics; we have used hand written C-code with AVX intrinsics as input to our tool. It is important to note that although gcc supports auto-vectorization, hand written AVX code is faster in 
most cases (Table 1). MMM and Diotgen are the most data dominant kernels and this is why they achieve the highest memory gains and speedup/energy gains on both CPUs. The proposed methodology achieves about $(8.5,30,2.1,2.5,2.3,3.8)$ times faster code, for (MMM, diotgen, MVM, FIR, Gemver, Gaussian Blur), on ZYBO and about $(4.4,3.9),(4,4),(20,2.3),(4,3.8),(1.3,1.2),(4.8,1.15)$ on i7 comparing to the (gcc, hand optimized code), respectively. Regarding energy gains, the proposed methodology achieves about $(9.2$, $40,2.5,2.7,2.6,5)$ times less energy on ZYBO and about $(4.6,4)$, $(4,4),(20,2.4),(3.7,3.6),(1.6,1.2),(4.8,1.15)$ on i7 comparing to the (gcc, hand optimized code), respectively. It is important to note that smaller gain values are achieved on $i 7$ processor because the input C-code contains AVX intrinsics, either directly or indirectly (gcc auto-vectorization). The proposed methodology achieves smaller gain values for SIMD input codes, because hand written AVX-code first, is at a lower level and thus more efficient, second, in many cases it already uses a significant number of the available registers, leaving less space for modifications and third, it is less friendly to register blocking.

Moreover, our methodology is evaluated over hand written optimized code and Polly [6] (Table 2). A large number of experiments has taken place with 10 different unroll factor values and 10 different loop tiling sizes in order to find the best (in Table 2, ' 1 loop' refers to best loop and best tile size). We have used normal C-code for ZYBO and hand written C-code using AVX instrinsics for i7. As it was expected, hand written optimized code achieves better or equal performance than gcc in all cases and likewise Table 1, our method achieves smaller gain values on the codes using AVX intrinsics. It is important to note that Polly includes other transformations too, which our methodology does not.

\section{CONCLUSION AND FUTURE WORK}

In this paper, a novel methodology to six of the most popular and important code optimizations is provided for data dominant static algorithms. Instead of applying heuristics and empirical methods we try to understand how software runs on the target HW and how $\mathrm{CO}$ affect ET and P. Moreover, we provide a theoretical model correlating the number of memory accesses and arithmetical instructions with CO parameters, HW parameters and input size. To this end, we make a first but important step towards correlating ET and $\mathrm{P}$ with $\mathrm{CO}, \mathrm{HW}$ architecture and input size.

Our future work includes more accurate and complex execution time models such as [22] as well as extending the P model to the remaining HW components. Moreover, it includes more loop transformations such as loop merge and loop distribution and considers nested loops where the array subscripts are not linear equations of the iterators.

\section{ACKNOWLEDGMENTS}

This work is partly supported by the European Commission under H2020-ICT-20152 contract 687584 - Transparent heterogeneous hardware Architecture deployment for eNergy Gain in Operation (TANGO) project.

\section{REFERENCES}

[1] Antonio Arts, Jos L. Ayala, Jos Huisken, and Francky Catthoor. 2013. Survey of Low-Energy Techniques for Instruction Memory Organisations in Embedded Systems. Signal Processing Systems 70, 1 (2013), 1-19. http://dblp.uni-trier.de/db/ journals/vlsisp/vlsisp70.html
[2] Wenlei Bao, Changwan Hong, Sudheer Chunduri, Sriram Krishnamoorthy, LouisNoël Pouchet, Fabrice Rastello, and P. Sadayappan. 2016. Static and Dynamic Frequency Scaling on Multicore CPUs. ACM Transactions on Architecture and Code Optimization (TACO) 13, 4 (2016).

[3] Nathan Binkert, Bradford Beckmann, Gabriel Black, Steven K. Reinhardt, Ali Saidi, Arkaprava Basu, Joel Hestness, Derek R. Hower, Tushar Krishna, Somayeh Sardashti, Rathijit Sen, Korey Sewell, Muhammad Shoaib, Nilay Vaish, Mark D. Hill, and David A. Wood. 2011. The Gem5 Simulator. SIGARCH Comput. Archit. News 39, 2 (Aug. 2011), 1-7. DOI : http://dx.doi.org/10.1145/2024716.2024718

[4] Uday Bondhugula, Albert Hartono, J. Ramanujam, and P. Sadayappan. 2008. A Practical Automatic Polyhedral Parallelizer and Locality Optimizer. SIGPLAN Not. 43, 6 (June 2008), 101-113. DOI : http://dx.doi.org/10.1145/1379022.1375595

[5] John Cavazos, Grigori Fursin, Felix Agakov, Edwin Bonilla, Michael F. P. O’Boyle, and Olivier Temam. 2007. Rapidly Selecting Good Compiler Optimizations Using Performance Counters. In International Symposium on Code Generation and Optimization (CGO '07). Washington, DC, USA, 185-197. DOI : http://dx.doi. org/10.1109/CGO.2007.32

[6] Tobias Grosser, Armin Größlinger, and Christian Lengauer. 2012. Polly - Performing Polyhedral Optimizations on a Low-Level Intermediate Representation. Parallel Processing Letters 22, 4 (2012). DOI: http://dx.doi.org/10.1142/ S0129626412500107

[7] John L. Hennessy and David A. Patterson. 2011. Computer Architecture, Fifth Edition: A Quantitative Approach (5th ed.). Morgan Kaufmann Publishers Inc., San Francisco, CA, USA.

[8] Vasilis Kelefouras, Georgios Keramidas, and Nikolaos Voros. 2017. Cache partitioning + loop tiling: A methodology for effective shared cache management. In IEEE Computer Society Annual Symposium on VLSI (ISVLSI 17). Bochum, Germany.

[9] DaeGon Kim, Lakshminarayanan Renganarayanan, Dave Rostron, Sanjay Rajopadhye, and Michelle Mills Strout. 2007. Multi-level Tiling: $M$ for the Price of One. In ACM/IEEE Conference on Supercomputing (SC). ACM, New York, NY, USA, Article 51, 12 pages. DOI : http://dx.doi.org/10.1145/1362622.1362691

[10] Prasad Kulkarni, Stephen Hines, Jason Hiser, David Whalley, Jack Davidson, and Douglas Jones. 2004. Fast Searches for Effective Optimization Phase Sequences. SIGPLAN Not. 39, 6 (June 2004), 171-182. DOI : http://dx.doi.org/10.1145/996893. 996863

[11] Prasad A. Kulkarni, David B. Whalley, Gary S. Tyson, and Jack W. Davidson. 2009. Practical Exhaustive Optimization Phase Order Exploration and Evaluation. ACM Trans. Archit. Code Optim. 6, 1, Article 1 (April 2009), 36 pages. DOI : http://dx.doi.org/10.1145/1509864.1509865

[12] Sameer Kulkarni and John Cavazos. 2012. Mitigating the Compiler Optimization Phase-ordering Problem Using Machine Learning. SIGPLAN Not. 47, 10 (Oct. 2012), 147-162. DOI : http://dx.doi.org/10.1145/2398857.2384628

[13] Hugh Leather, Edwin Bonilla, and Michael O'Boyle. 2009. Automatic Feature Generation for Machine Learning Based Optimizing Compilation. In 7th Annual IEEE/ACM International Symposium on Code Generation and Optimization (CGO). Washington, USA, 81-91. DOI : http://dx.doi.org/10.1109/CGO.2009.21

[14] Sheng Li, Jung Ho Ahn, Richard D. Strong, Jay B. Brockman, Dean M. Tullsen, and Norman P. Jouppi. 2009. McPAT: An Integrated Power, Area, and Timing Modeling Framework for Multicore and Manycore Architectures. In 42Nd Annual IEEE/ACM International Symposium on Microarchitecture (MICRO 42). ACM, 469480. DOI : http://dx.doi.org/10.1145/1669112.1669172

[15] Jun Liu, Yuanrui Zhang, Wei Ding, and Mahmut T. Kandemir. 2011. On-chip cache hierarchy-aware tile scheduling for multicore machines.. In International Symposium on Code Generation and Optimization. 161-170. http://dblp.uni-trier. de/db/conf/cgo/cgo2011.html

[16] Martin Palkovic, Francky Catthoor, and Henk Corporaal. 2009. Trade-offs in loop transformations. ACM Trans. Design Autom. Electr. Syst. 14, 2 (2009). DOI : http://dx.doi.org/10.1145/1497561.1497565

[17] Louis-Noël Pouchet, Uday Bondhugula, Cédric Bastoul, Albert Cohen, J. Ramanujam, P. Sadayappan, and Nicolas Vasilache. 2011. Loop Transformations: Convexity, Pruning and Optimization. SIGPLAN Not. 46, 1 (Jan. 2011), 549-562. DOI : http://dx.doi.org/10.1145/1925844.1926449

[18] Dan Quinlan, You Haihang, Yi Qing, Richard Vuduc, and Keith Seymour. 2007. POET: Parameterized Optimizations for Empirical Tuning. IEEE International Parallel and Distributed Processing Symposium (2007).

[19] Lakshminarayanan Renganarayanan, DaeGon Kim, Sanjay Rajopadhye, and Michelle Mills Strout. 2007. Parameterized Tiled Loops for Free. SIGPLAN Not. 42, 6 (June 2007), 405-414. DOI : http://dx.doi.org/10.1145/1273442.1250780

[20] Michele Tartara and Stefano Crespi Reghizzi. 2013. Continuous learning of compiler heuristics. ACM Trans. Archit. Code Optim. 9, 4, Article 46 (Jan. 2013), 25 pages. DOI : http://dx.doi.org/10.1145/2400682.2400705

[21] Ohio State University. 2012. PolyBench/C benchmark suite. (2012). http://web. cs.ucla.edu/ pouchet/software/polybench/

[22] Dawei Wang and Xian-He Sun. 2014. APC: A Novel Memory Metric and Measurement Methodology for Modern Memory Systems. IEEE Trans. Comput. 63, 7 (July 2014), 1626-1639. DOI : http://dx.doi.org/10.1109/TC.2013.38 